7. Reprod. Fert. (1966) 11, 371-377

\title{
FLUCTUATIONS IN THE SWIMMING RATE OF INDIVIDUAL BULL SPERMATOZOA
}

\author{
DOREEN TAMPION* \\ National Institute for Research in Dairying, \\ Shinfield, Reading, Berks.
}

(Received 14th September 1965)

\begin{abstract}
Summary. Individual bull spermatozoa were tracked over a distance of several millimetres, measuring as often as possible the time required to swim the length of the micrometer $(310 \mu)$.

In oestrous mucus from the cow's uterine cervix, the rates of some spermatozoa were found to fluctuate widely, while others remained constant for long distances. Homogeneity of the medium was tested by comparing mean rates of groups of spermatozoa in different regions of the mucus. The material was not sufficiently heterogeneous to account for the results. The timing error could account for only a small part of the variation.

Similar fluctuations in swimming rate were observed in saline and in the supernate obtained by spinning off spermatozoa from a $20,000 / \mu \mathrm{l}$ suspension in saline.
\end{abstract}

\section{INTRODUCTION}

In discussions of the swimming rate of spermatozoa, it has usually been tacitly assumed that the rate for an individual spermatozoon is constant, and that a given sample of semen contains a mixed population in which some spermatozoa have intrinsically higher rates than others.

Variability in swimming rates in a given population could also theoretically be accounted for if all the spermatozoa had the same swimming capacity, but some or all swam at a variable rate.

According to Rikmenspoel (1957), plotted spermatozoa tracks from films of spermatozoa movement indicate that rate is uniform for a given spermatozoon. Other workers have also measured spermatozoa swimming rates from films, and have not described individual rates as variable (Rothschild, 1953; Gray, 1958). However, the size of the field normally used in microphotography is rather small, and might obscure occasional abrupt changes of rate if these alternated with periods of steady swimming, unless very large numbers of spermatozoa tracks were examined.

The following observations of individual live spermatozoa are the result of a direct approach to this problem. The stop-watch method of rate estimation (Tampion \& Gibbons, 1963a) can be used to time successive rates of the same

\footnotetext{
* Present address: Balmore Cottage, Hemdean Road, Reading.
} 
spermatozoon over a considerable distance, but only if a sufficiently dilute spermatozoal suspension is used. In addition, the spermatozoa must be moving slowly to allow manipulation of the mechanical stage and focus, and in order to manoeuvre the spermatozoon back to the starting point of the eyepiece micrometer after each estimation of swimming rate. A good medium for this purpose is the uterine cervical mucus of the cow, since it both slows down the spermatozoa and permits study of them at very high dilutions (Tampion \& Gibbons, 1963b). Further, a thread of mucus orientates the spermatozoa in parallel straight lines (Tampion \& Gibbons, 1962), which greatly facilitates tracking. It was possible to follow individual spermatozoa for a distance of several millimetres in mucus. This medium has the great advantage of being a natural environment in which active spermatozoa would normally become highly diluted in vivo. A possible disadvantage of the medium is the apparent inhomogeneity, which might induce variations in velocity. For this reason further spermatozoa 'tracks' were also measured in the less satisfactory but homogeneous medium made by spinning off spermatozoa from a 20,000/ $\mathrm{l}$ suspension in saline ('sperm extract').

\section{MATERIALS AND METHODS}

The mucus was taken from cows at oestrus and frozen until use. For the experiments with mucus, a rectangular frame cut from polyethylene sheet $\frac{1}{32}$ in. thick was dipped in melted Vaseline petroleum jelly and applied to a microscope slide. A thread of mucus, drawn out with a platinum hook, was laid within the rectangle and a drop of semen was added at one end. A coverslip was then pressed down on top to make an airtight chamber, with the mucus adhering to the coverslip. The preparation was examined by means of a microscope maintained at $37^{\circ} \mathrm{C}$, after allowing a period of $5 \mathrm{~min}$ for temperature equilibration. An individual spermatozoon was then picked out in the region of mucus into which few had as yet penetrated, and followed over a path of several millimetres, measuring as often as possible the time taken to swim the length of the eyepiece micrometer, a distance of $310 \mu$ at the magnification used.

Since the use of an ordinary stop-watch presented difficulties, the following arrangement was adopted for timing spermatozoa. An electrically controlled stop-clock was started and stopped by a lever held in the left hand of the observer while an assistant recorded times and then immediately reset the clock to zero.

The 'timing error' was estimated by using the stop-clock to time the movement of the seconds' hand of an electric wall clock. Means and standard deviations were prepared for five time intervals, with twenty observations at each interval. The percentage error of the mean varied from 0.0 to $1.1 \%$. The coefficients of variation ranged from $3.01 \%$ to $6.33 \%$. The arrows on the diagrams show the $95 \%$ confidence limits of each corresponding rate.

\section{Spermatozoa tracks in mucus}

\section{RESULTS}

Text-fig. 1. shows two results from each of five experiments with mucus. Each bar represents a measured rate, i.e. the mean rate over a distance of $310 \mu$. The difficulty of getting the spermatozoon back to the 'starting point' 
each time made it impossible to standardize the time (or distance) between successive rates, so that the spacing of the bars along the abscissa is arbitrary.

When these figures were analysed statistically, it was found that within some of the spermatozoa tracks the variation in rate was not significantly less than the variation in rate between ten separate spermatozoa. Only a small proportion of the variation in individual spermatozoa could be accounted for by the timing error.
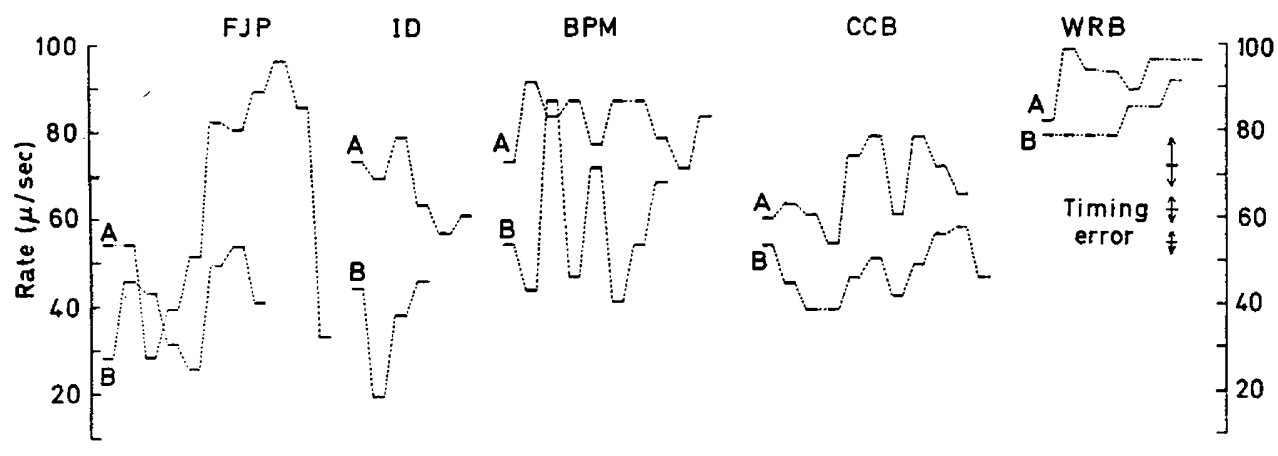

Successive time intervals

TEXT-FIG. 1. Fluctuations of swimming rate in mucus. Two examples from each of five bulls.

In all five samples of semen examined, some spermatozoa were found to be swimming at an approximately constant rate (e.g. WRB, B), while others showed wide fluctuations (e.g. FJP, A). Variation in rate was in fact greater than could be recorded by this technique, since within the distance of $310 \mu$ over which the rate was measured, spermatozoa could occasionally be seen to decelerate abruptly, stop for a fraction of a second and then continue with rapid acceleration.

\section{Homogeneity of mucus}

The rates of groups of ten spermatozoa were measured in several different regions of three samples of mucus to test for homogeneity. For one sample of mucus the mean rates were $50 \cdot 7,55 \cdot 8,57 \cdot 3,61.3$ and $62.0 \mu / \mathrm{sec}$, for a second sample: $60 \cdot 0,66 \cdot 2,68 \cdot 0,73 \cdot 1$ and $77 \cdot 6 \mu / \mathrm{sec}$, and for the third sample: $50 \cdot 3$, $52 \cdot 9,58 \cdot 3,63 \cdot 7,68 \cdot 6,72 \cdot 2,72 \cdot 3$ and $73.5 \mu / \mathrm{sec}$. Although these variations are considerable, and differences between the extremes are significant, the differences are much less than the fluctuations within some of the individual spermatozoa. Inhomogeneity of the mucus cannot, therefore, account for all the observed fluctuations in rate.

Gross inhomogeneity is sometimes apparent in a sample of mucus in that there may be a thread, perhaps $1 \mathrm{~mm}$ wide, from which spermatozoa are completely absent, when many are swimming in the remainder of the preparation. Such complete discontinuities are, of course, irrelevant to the present problem. However, within a region of the order of a millimetre in which spermatozoa are present, they may appear to be distributed in definite pathways, as previously described (Tampion \& Gibbons, 1963b). If this behaviour 
indicated minute threads within the mucus which were too viscous to be penetrated at all, then considerable differences in viscosity of the penetrable mucus would also be expected. The following observations were made to test this possibility. The eyepiece micrometer was arranged at right angles to a stream of spermatozoa swimming in mucus, and the point at which each cell crossed it was recorded. After a short period of time the spermatozoa seemed concentrated at certain crossing places, while none were observed in other regions of the scale. But if the preparation was examined for long enough, new columns of spermatozoa came in at different points, until eventually the crossing places were randomly distributed all along the scale. Epithelial cells in the mucus remained stationary during observation, so that there was no question of shifting or flowing of the field. The strain-induced structure in the mucus would seem, therefore, to keep the spermatozoa together in orientated groups, but not to determine the actual course of swimming.

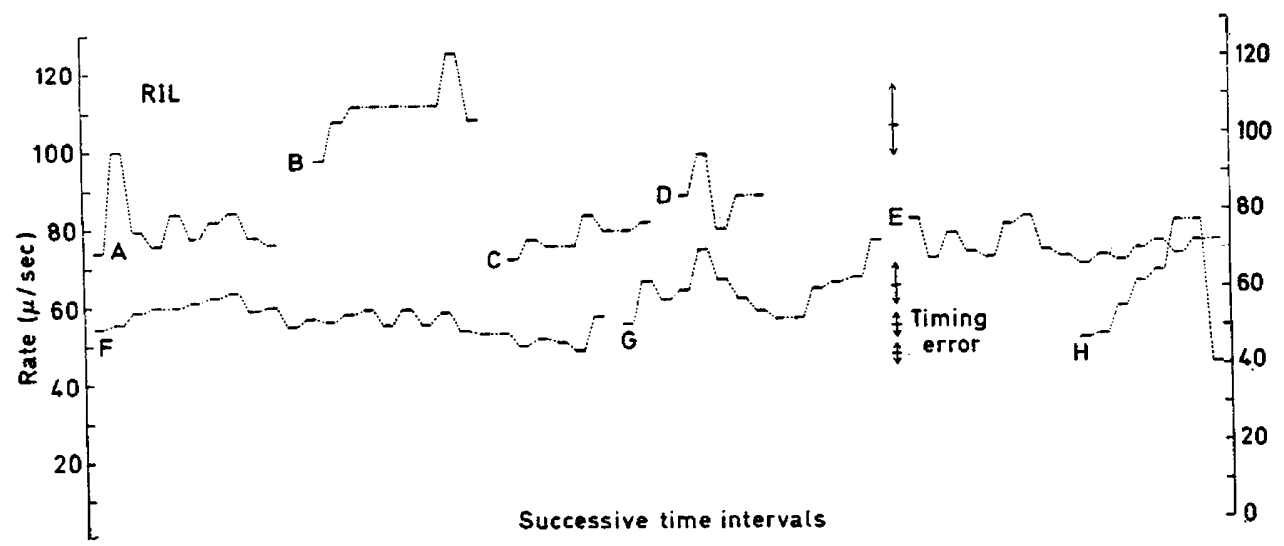

Text-FiG. 2. Fluctuations of swimming rate in 'sperm extract'. Individual spermatozoa from the bull RIL.

\section{Spermatozoa tracks in 'sperm extract'}

The best results in this medium were obtained for the bull RIL at a spermatozoal concentration of $1000 / \mu \mathrm{l}$ (Text-fig. 2). Spermatozoa from some other bulls swam too fast ( $150 \mu / \mathrm{sec}$ or more) even at this dilution, and could not be followed. Tracks of spermatozoa from four other bulls were sufficiently slowed down by these conditions. Although rates in 'sperm extract' were on the whole higher than in mucus and the timing error correspondingly larger, some of the fluctuations were much greater than could be accounted for by timing error. Some of the spermatozoa were, however, travelling at a remarkably steady pace. Track F of RIL (Text-fig. 2) for example, which consists of twenty-seven measurements, representing a distance of more than $1 \mathrm{~cm}$ and a time period of over $2 \frac{1}{2} \mathrm{~min}$, shows little change over a distance of about $6 \mathrm{~mm}$ and during more than a minute. Track $H$ (RIL) on the other hand practically doubled and later halved its rate in about $3 \mathrm{~mm}$, corresponding to about $\frac{3}{4} \mathrm{~min}$.

Considerable variation in manner of swimming was also observed in the course of tracking. Some spermatozoa travelled over a distance of several millimetres without changing course appreciably. Others swerved, sometimes a 
little to the left, sometimes to the right, so that the general direction remained the same. A number of spermatozoa in all preparations travelled in a way which could be loosely described as 'circling', but which more nearly resembled tracing out a polygon, since such spermatozoa swam nearly straight for a little distance, then swerved slightly, but always to the same side, so that complete circuits were eventually performed. Spermatozoa, behaving in this way, were seen to continue 'circling' in the same sense for many circuits without a change of direction. These spermatozoa were rotating in the same manner as those swimming in straight lines and are not to be compared with the layer of spermatozoa immediately below the coverslip, whose heads are held constantly parallel to the glass (Tampion \& Gibbons, 1963b).

In four of these experiments approximately 100 cells in each preparation were classified according to movement, and it was found that, on average, $71 \%$ of spermatozoa swam straight over a distance of more than $310 \mu$ (range $56 \%$ to $90 \%) ; 7 \%$ swerved to right and left $(4 \%$ to $10 \%)$, while the remainder swam in polygonal patterns.

The side of the polygon described by 'circling' spermatozoa may be $0.5 \mathrm{~mm}$ or more, in which case such cells would be included in velocity determinations, and in fact would be indistinguishable from 'straight' spermatozoa under normal conditions of velocity measurement, unless the swerve came in the middle of the micrometer. The mean rates of 'straight' and 'circling' cells would not necessarily be the same, even if it is true, as seems probable, that the former are simply tracing out a much larger circle or polygon than the latter. This point was checked by comparing the rate of spermatozoa which swam along the micrometer and so could be timed, but which swerved to one side before disappearing from view (i.e. in the next $\frac{1}{3} \mathrm{~mm}$ ), with those which swam straight across the entire field. The mean rates of straight and circling spermatozoa were estimated for four ejaculates; the mean of the means for the former was $133.4 \mu / \mathrm{sec}$ and for the latter $134.0 \mu / \mathrm{sec}$. The size of the polygonal track of the spermatozoa seems, therefore, unrelated to velocity, provided that the sides are at least $\frac{1}{3} \mathrm{~mm}$.

Generally it is not possible to follow an individual spermatozoon in saline, since a sufficiently high dilution in this medium would be lethal. However, one sample of semen (another ejaculate of the bull RIL) which had been diluted to 5000 spermatozoa/ $\mu$ l with saline, was found to be very suitable for such measurements, as a large proportion of the spermatozoa had died and fallen out of sight, leaving relatively few in the microscope field, but these few were swimming normally, $80 \%$ of them in straight lines. Five fairly long tracks were obtained in which the rates varied (a) from 60.8 to $75.6 \mu / \mathrm{sec}$, (b) from 58.5 to $63.2 \mu / \mathrm{sec}$, (c) from 57.4 to $83.8 \mu / \mathrm{sec}$, (d) from 64.6 to $94.0 \mu / \mathrm{sec}$ and (e) from 62.0 to $103.3 \mu / \mathrm{sec}$. The fluctuations in this medium were therefore as remarkable as in the other two media.

\section{DISCUSSION}

It appears from these data that the swimming behaviour of bull spermatozoa is more complicated than it would seem to be at first sight. In a given population 
there may be spermatozoa swimming at uniformly high or low rates, and also cells whose velocities change considerably from time to time. While one cannot be sure, on the basis of these results, that the activity of spermatozoa in whole semen, or other media at other concentrations, would also fluctuate, it is certain that variable behaviour can be observed in a variety of circumstances, and this must be taken into account when interpreting experiments. This implies, for instance, that any comparatively simple correction for a velocity selection error, as discussed in a previous publication (Tampion \& Gibbons, 1963a) and as applied by Rikmenspoel (1957) to his results is not appropriate. If all spermatozoa had a variable rate no correction at all would be needed, but if, as seems to be the case, the rate is uniform in some spermatozoa but not in others, then no universal correction could be applied, since the proportion uniform and degree of fluctuation would vary from one experiment to the next.

Changes in the mean velocity can conveniently be used as a measure of the effects of various treatments (e.g. the effect of $\mathrm{pH}$; Tampion \& Gibbons, 1963a). In the light of these results for individual spermatozoa, changes in mean velocity may have several different interpretations. A decrease in mean velocity, for instance, might imply that all the spermatozoa had been slowed down, or that the length of 'slow phase' had been increased relative to the length of 'fast phase' for individual spermatozoa, or that spermatozoa formerly swimming at a uniformly high rate had developed periodic slow phases. Undoubtedly, further investigation of this phenomenon should throw some light on the nature of normal senescence and artificial inhibition of spermatozoa.

This variation in swimming rate is not confined to bull spermatozoa. Boar spermatozoa can be seen to accelerate, slow down, stop and start again, but so far no satisfactory measurements of their fluctuations in rate have been obtained. Long-term tracking of spermatozoa of this species is almost impossible because of their erratic movements (Tampion, 1963). Ritchie (1950), while studying human spermatozoa by stroboscopic light, noticed that the cells did not move forward at a steady rate. Gray (1955) has also remarked that spermatozoa of the sea-urchin Psammechinus miliaris stop and start again while under observation. This implies change of spermatozoa velocity in their normal environment (sea-water) at a dilution which is stimulating rather than damaging. In this species at least, it can, therefore, be said with certainty that rate is not constant under conditions obtaining in nature.

\section{ACKNOWLEDGMENTS}

I should like to give thanks to the staff of the Reading Cattle Breeding Centre for their generous help, and also to Miss J. Davies, who gave able technical assistance. This work was financed by the Population Council Inc., New York, whose help is gratefully acknowledged.

\section{REFERENCES}

GRAY, J. (1955) The movement of sea-urchin spermatozoa. F. exp. Biol. 32, 775.

GRAY, J. (1958) The movement of the spermatozoa of the bull. F. exp. Biol. 35, 96.

RIKmenspoel, R. (1957) Photoelectric and cinematographic measurements of the 'motility' of bull sperm cells. Thesis, University of Utrecht. 
RrTchis, D. (1950) The frequency of beat of sperm tails. Science, N.Y. 111, 172.

RotHSCHILD, LORD (1953) A new method of measuring the activity of spermatozoa. F. exp. Biol. 30, 178.

TAMPION, D. (1963) Swimming rate of boar spermatozoa. F. Reprod. Fert. 6, 471.

TAMpion, D. \& GrbBons, R. A. (1962) Orientation of spermatozoa in mucus of the cervix uteri. Nature, Lond. 194, 381.

TAMPion, D. \& Gribons, R. A. (1963a) Effect of $\mathrm{pH}$ on the swimming rate of bull spermatozoa. 7. Reprod. Fert. 5, 249.

TAmpion, D. \& Gibbons, R. A. (1963b) Swimming rate of bull spermatozoa in various media and the effect of dilution. F. Reprod. Fert. 5, 259. 